\title{
Gal-3 y ST2 como biomarcadores: un paso al frente en el pronóstico de la Insuficiencia Cardíaca
}

\author{
Gal-3 and ST2 Biomarkers - A Step Forward in Heart Failure Prognostication
}

\author{
Mário Barbosa, MD, MSc ${ }^{1}$, Andreia Matos, MSc ${ }^{2,3,4,5}$, Manuel Bicho, MD, PhD ${ }^{2,3,}$ Luiz Menezes Falcão, MD, PhD ${ }^{6,7}$ \\ ${ }^{1}$ Department of Internal Medicine, Hospital Lusíadas Lisboa, Lisbon, Portugal. "2Genetics Laboratory and Environmental Health Institute-ISAMB, Lisbon, Portugal. ${ }^{3 / n s t i t u t o}$ \\ de Investigação Científico Bento da Rocha Cabral, Lisbon, Portugal. ${ }^{4}{ }^{3}$ S-Instituto de Investigação e Inovação em Saúde/INEB-Institute of Biomedical Engineering, University \\ of Porto, Porto, Portugal. ${ }^{5} \mathrm{Abel}$ Salazar Institute for the Biomedical Sciences (ICBAS), University of Porto, Portugal. ${ }^{6}$ Department of Internal Medicine, Santa Maria Hospital, \\ Lisbon, Portugal. ' 'Lisbon Faculty of Medicine, Lisbon, Portugal.
}

\begin{abstract}
Aims: The American College of Cardiology (ACA)/ American Heart Association (AHA) granted Galectin-3 (Gal-3) and Suppression of Tumorigenicity 2 (ST2) evaluation a class II recommendation for HF prognosis, as an adjunctive to conventional clinical risk factors and natriuretic peptides dosing in 2013. However, in Europe this endorsement is not valid. The purpose of this study was to study the association of Gal-3 and ST2 collected at-admission with early (defined as the period of 90 days post-discharge) rehospitalization and overall mortality, and end of follow-up overall mortality in HF patients. Additionally, aminoterminal B-type natriuretic peptide (NT-proBNP) at admission was considered to test if a multi-marker strategy could yield supplementary information.

Material and Methods: Gal-3, ST2 and NT-proBNP were assessed in patients hospitalized with acute decompensated HF in class III or IV of New York Heart Association (NYHA). Univariate Cox proportional hazard model was used to assess the relationship between variables and outcomes. Since there are no standardized cut-offs for Gal-3 and ST2, the multiclass Area Under the Curve Receiver-Operator Characteristic (AUCROC) as defined by Hand and Till was used to evaluate the overall performance of each biomarker as a predictor of the outcomes.

Results: We followed 65 patients for a median of 13.7 (Q1-Q3 6.7-18.9) months. Gal-3 correlated with short-term rehospitalization (HR: 9.886, 95\% Cl: 2.027-48.214, P-value=0.005), short-term mortality (HR: 13.731, 95\% Cl: 1.650-114.276, P value=0.015) and end of follow-up mortality (HR: 4.492, 95\% Cl: 1.594-12.656, P-value=0.004). The association of elevated NT-proBNP determinations increased the risk of short-term rehospitalization (HR: 11.985, 95\% Cl: 1.962-73.218, P value=0.007) and end of follow-up mortality (HR: 78.025, 95\% Cl: 7.592-801.926, P-value<0.001). ST2 correlated with end of follow-up mortality (HR: 4.846, 95\% Cl: 1.396-16.825, P-value=0.013). The risk further increased if NT-proBNP (HR: 5.953, 95\% Cl: $1.683-$ 21.055, P-value $=0.006$ ) or Gal-3 determinations (HR: 6.209, 95\% Cl: 2.393-16.114, P-value<0.001) were added.

Conclusions: Elevated Gal-3 concentrations correlated with short-term rehospitalization, short-term mortality and end of follow-up mortality; whereas ST2 prognosticated end of follow-up mortality. Collective analysis with elevated NT-proBNP values further increased the outcomes' risk. These results corroborate the assumption that promising novel biomarkers Gal-3 and ST2 could be valuable for HF risk stratification. We highlight that a multi-marker strategy added information, as a synergism between myocardial fibrosis biomarkers and the myocardial stretch peptide was observed.

Key Words: Acute decompensated heart failure; Short-term readmission; Short-term mortality; Galectin-3; ST2; NT-proBNP.
\end{abstract}

\section{INTRODUCTION}

Despite scientific advances HF short-term prognosis remains poor, as circa $25 \%$ of patients are readmitted in the first 30 days after hospital discharge ${ }^{1}$ and approximately $30 \%$ are readmitted 60 to 90 days post-discharge 2 .

Remarkably, the mortality rate 60 to 90 day post-discharge reaches $15 \%$.

It urges to define a short-term prognosis for HF patients in order to reduce readmission and premature mortality.

The actual treatment paradigm of $\mathrm{HF}$ is based, fundamentally, on the blockade of the renin-angiotensin-aldosterone system, nevertheless other mechanisms influence the genesis and progression of this syndrome.

The need for risk markers in precocious stages of the disease, in order to refrain its evolution, has led to an increasing interest in biomarkers related to the physiopathological pathways of $\mathrm{HF}$, namely myocardial fibrosis. Myocardial fibrosis seems to favor left ventricular dysfunction which determines HF progression ${ }^{3}$.

Clinical judgement, the cornerstone of medical praxis is enhanced by imaging and biological diagnostic tests.
Fibrosis biomarkers emerge as promising tools that can complement traditional clinical practice by recognizing high risk patients that could benefit from therapeutic intensification and stricter surveillance.

The role of natriuretic peptides as diagnostic predictors is undisputed ${ }^{4}$ but Gal- $3^{5,6}$ and ST2 ${ }^{7,8}$ seem to be superior concerning prognosis.

In 2013, the ACA/ AHA recognized ST2 and Gal-3 as HF prognosticators, ${ }^{9}$ nevertheless the European Society of Cardiology (ESC) does not support this recommendation ${ }^{10}$. Interestingly, a multi-marker strategy appears to yield additional information, as current data alludes a synergism between myocardial stretch peptides and fibrosis biomark$\operatorname{ers}^{4,5,6,11 .}$

\section{MATERIAL AND METHODS}

Study design and population

The pREdictors of Early REadmission iN Chronic hEart failure (REFERENCE) was an observational prospective cohort, single-center, single-arm study. 
Patients were recruited consecutively for a period of 12 months from an Internal Medicine ward.

Inclusion criteria were age $\geq 18$ years old and hospitalization due to chronic decompensated HF patients in class III or IV of NYHA.

Exclusion criteria were:

1. in-hospital death in the first hospitalization;

2. hospital discharge against medical advice;

3. chronic kidney disease patients with a glomerular filtration rate (GRF) $<30 \mathrm{ml} / \mathrm{min} / 1.73 \mathrm{~m}^{2}$ (calculated with the Modification of Diet in Renal Disease score) or under renal replacement therapy;

4. moderate or severe hepatic impairment (calculated with the Child-Pugh score);

5. active neoplasm with or without metastasis.

The participation in the study was entirely voluntary and all eligible subjects signed the written informed consent.

The study was performed in conformity with its protocol, the Declaration of Helsinki and the Oviedo Convention. The study was approved by an Institutional Review Board (Academic Medical Center Ethics Committee).

\section{Protocol and definitions}

The diagnosis of HF was based on the ESC guidelines ${ }^{10}$.

Patient evaluation followed study protocol that included clinical history, physical examination, 12-lead electrocardiogram, thoracic X-ray, blood sampling for laboratory tests, transthoracic Doppler echocardiography and therapeutic data.

All echocardiograms M mode, two-dimensional and Doppler were performed by an experienced operator using a Hitachi Aloka alfa 6 Medical device with a $2.5 \mathrm{MHz}$ transducer.

Biochemical parameters were assessed as per routine using plasma samples.

Biomarkers were measured using plasma samples.

Plasma samples were stored at the study sites at $-20^{\circ} \mathrm{C}$, followed by storage at $-80^{\circ} \mathrm{C}$.

Galectin-3 and ST2/IL-33R were quantified in plasma using pre-coated human ELISA R\&D Systems, Abingdon, USA kits. Plasma NT-proBNP values were measured using electrochemiluminescence immunoassay Elecsys NT-proBNP Roche Diagnostics, GmbH, Mannheim, Germany.

In light of the European Society of Cardiology guidelines, HF with preserved ejection fraction (HFpEF) was defined as left ventricular ejection fraction (LVEF) $\geq 50 \%$, midrange ejection fraction (HFmrEF) was defined as LVEF 40-49\% and reduced ejection fraction (HFrEF) was defined as LVEF $<40 \% .{ }^{10}$

\section{Outcomes}

We studied the correlation of Gal-3 and ST2 with short-term rehospitalization due to $\mathrm{HF}$, short-term all-cause mortality and end of follow-up all-cause mortality in HF patients.
Short-term rehospitalization was defined as rehospitalization within 90 days of hospital discharge.

Short-term mortality was defined as death occurring within 90 days after hospital discharge.

End of follow-up mortality was defined as death that occurred during the entire study period.

\section{Statistical analysis}

Convenience sampling was used and no sample size calculation was performed. Categorical variables were summarized by relative and absolute frequencies and compared using the chi-squared test or Fisher's Exact test.

Continuous variables were summarized by mean, standard deviation, median, first and third quartiles (Q1-Q3). Shapiro-Wilk test was used to assess the normality of continuous variables. Comparisons between patients with or without an event of interest were performed using the t-test or Wilcoxon Rank test, as applicable.

Short-term rehospitalization, short-term mortality and end of follow-up mortality were considered as stratification variables.

A survival analysis was performed for the events of interest. For each endpoint, Kaplan-Meier survival estimates were calculated and plotted for each categorical variable. Logrank tests were used to compare survival probabilities in each of the considered variables. A univariate Cox proportional hazards model was fitted to the data to obtain HR and $95 \% \mathrm{Cl}$ for each variable.

The proportional hazards assumption was tested using Schoenfeld residuals. All analyses were conducted at an overall significance level of $5 \%$. No imputation was performed on missing data. No adjustments for multiplicity were performed.

Since there are no standardized cut-offs for Gal-3 and ST2, the multiclass AUCROC as defined by Hand and Till was used to evaluate the overall performance of each biomarker as a predictor of the outcomes.

Taking into consideration the low number of subjects, it was decided to consider as relevant predictors biomarkers that had an AUCROC above 0.7 and in which the 95\% confidence interval did not contain 0.5 (value for which the predictor makes random guesses). The optimal cut-off value for each biomarker to predict each of the events was defined using the Youden Index, which maximizes the sensitivity and specificity of the predictor. Negative predictive values (NPV) and positive predictive values (PPV) were calculated and reported for the selected cut-offs.

\section{RESULTS}

From the 70 selected patients 5 were excluded from the analysis given that during follow-up they were diagnosed with active neoplasm.

The 65 patients who matched the selection criteria had a median follow-up period of 13.7 (6.7-18.9) months. 
The mean age of the patients was $79.2 \pm 10.8$ years, $56.9 \%$ were female and their mean LVEF was $50.38 \pm 19.07 \%$.

Baseline characteristics of the population study are presented in Table 1.

The 90-day post-discharge readmission percentage was $33.8 \%$ and the 90 -day mortality was $18.5 \%$. By the end of follow-up $40 \%$ of the patients had died.

\section{Spearman's correlation coefficient}

Spearman's correlation coefficient recognized an inversely proportional correlation between Gal-3 levels and baseline glomerular filtration rate (GFR) (Coefficient: -0.537, P-value $<0.001$ ) and admission GFR (Coefficient: -0.549, P-value $<0.001$ ).

In contrast, Spearman's correlation revealed that Gal-3 and NT-proBNP values were proportional (Coefficient: 0.383, P-value $=0.009$ ), as depicted in Figure 1.

The same statistical method showed a significant negative correlation between ST2 levels and baseline GFR (Coefficient: -0.418 , P-value $<0.001$ ) and admission GFR (Coefficient: -0.438 , P-value $<0.001$ ).

Figure 1. Spearman correlation of Galectin-3 and NT-proBNP

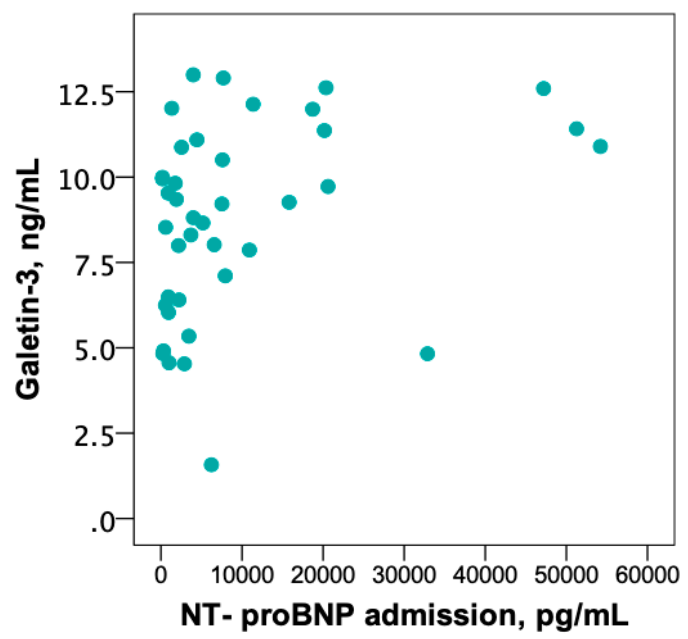

$(r=0.383, P$-value $=0.009)$

\section{ROC analysis}

Regarding ROC analysis, for the short-term rehospitalization outcome, only Gal-3 had an acceptable AUCROC value (AUCROC: 0.74; CI95\%: 0.55-0.92). For this biomarker, the optimal cut-off value (Youden Index) was $11.41 \mathrm{ng} / \mathrm{mL}$ with a high NPV (0.93) indicating a good capability in predicting (93\% probability) that no short-term rehospitalization events occur if the biomarker is below the cut-off. The PPV was 0.54 , being able to predict the event with a probability of $54 \%$.

Concerning the short-term mortality outcome, there were two biomarkers with acceptable AUCROC values, Gal-3 (AUCROC: 0.85; CI95\%: 0.74-0.97) and NT-proBNP (AUCROC: 0.75; Cl95\%: 0.58-0.92).

For Gal-3, the optimal cut-off value was $10.98 \mathrm{ng} / \mathrm{mL}$ with a perfect NPV (1.0), indicating an excellent capability in predicting (100\% probability) that no short-term mortality events occur if the biomarker is below the cut-off. Regarding the capability of predicting short-term mortality events, the biomarker had inferior capabilities (PPV: 0.44), but it is still relevant enough to take it into consideration, given the $44 \%$ probability of the event to occur.

For NT-proBNP the optimal cut-off value was $21336 \mathrm{ng} / \mathrm{L}$ with a high NPV (0.91). The PPV was inferior (0.64), being able to predict with $64 \%$ probability an event of short-term death.

Minding the end of follow-up mortality outcome, we decided to consider Gal-3 and ST2 although they had an AUCROC slightly below 0.7 [Galectin-3 (AUCROC: 0.69; CI95\%: 0.520.86) and ST2 (AUCROC: 0.69; Cl95\%: 0.53-0.85)], due to the fact that they had a significant behavior ( $95 \%$ confidence intervals above 0.5).

For Gal-3 the optimal cut-off value was $9.99 \mathrm{ng} / \mathrm{mL}$ with a NPV of 0.78 and PPV of 0.65 .

The ST2 biomarker had similar capabilities to Gal-3, with an optimal cut-off value of $24.79 \mathrm{ng} / \mathrm{Lwith}$ NPV of 0.83 and a PPV of 0.60 .

Figure 2. Short-term mortality - Kaplan Meier: GAL3 $\geq 10.97$ ng/mL

GAL3 $>=10.97 \mathrm{ng} / \mathrm{mL}+$ № + Yes

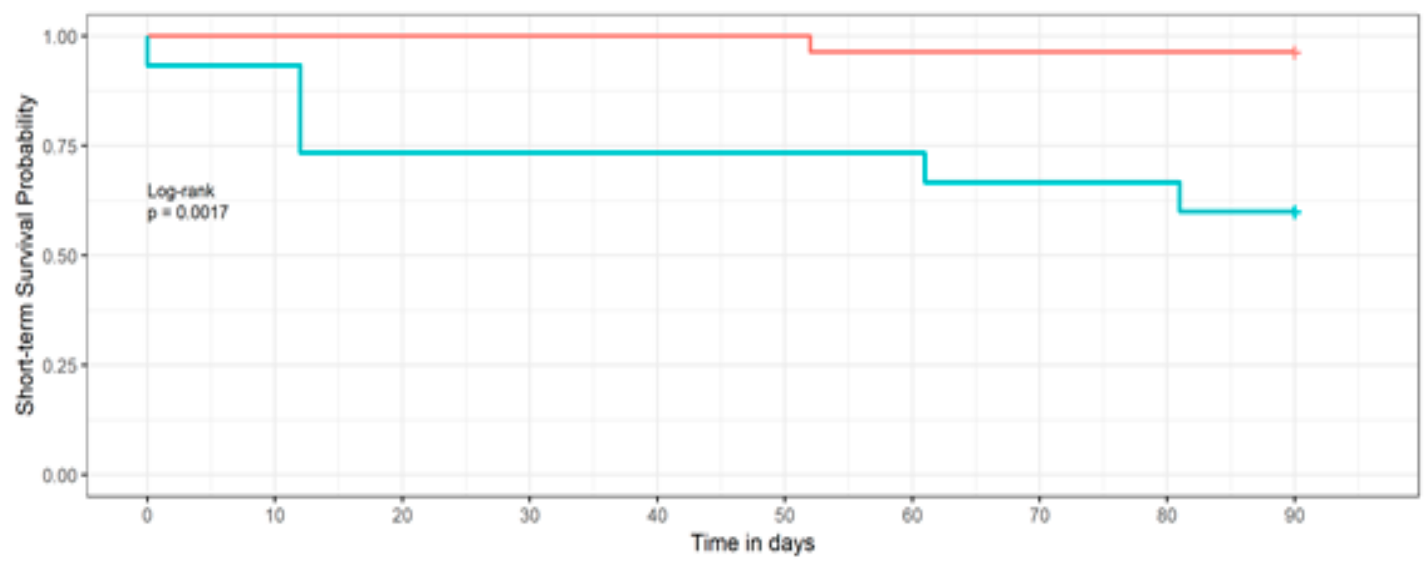


Table 1. Baseline characteristics of the population study

\begin{tabular}{|c|c|}
\hline Characteristics & Patients $(n=65)$ \\
\hline Age, mean (SD) & $79.2 \pm 10.8$ \\
\hline Female Gender, $\mathrm{n}(\%)$ & $37(56.9)$ \\
\hline Hypertension, $n(\%)$ & $58(89.2)$ \\
\hline Type 2 Diabetes, n (\%) & $25(38.5)$ \\
\hline Dyslipidemia, $n(\%)$ & $41(63.1)$ \\
\hline Obesity, n (\%) & $17(26.2)$ \\
\hline Atrial Fibrillation, $\mathrm{n}(\%)$ & $28(43.1)$ \\
\hline Family History of CVD, $\mathrm{n}(\%)$ & $31(47.7)$ \\
\hline Tabagism, n (\%) & $21(32.3)$ \\
\hline Chronic Kidney Disease, n (\%) & $34(52.3)$ \\
\hline GFR (Baseline), median & $57.8(43.8-82.2)$ \\
\hline GFR (Admission), median & $47.9(33.2-68.1)$ \\
\hline Previous Acute Myocardial Infarction, $n(\%)$ & $27(41.5)$ \\
\hline Hypertensive Heart disease, $\mathrm{n}(\%)$ & $44(67.7)$ \\
\hline Ischemic Heart disease, $\mathrm{n}(\%)$ & $22(33.8)$ \\
\hline Valvular Heart disease, $n(\%)$ & 56 (86.2) \\
\hline LVEF, mean (SD) & $50.38 \pm 19.07$ \\
\hline NYHA class III, $n(\%)$ & 43 (66.2) \\
\hline ACE Inhibitor, $\mathrm{n}(\%)$ & $43(66.2)$ \\
\hline Beta Blocker, n (\%) & $38(58.5)$ \\
\hline Mineralocorticoid Receptor Antagonists, n (\%) & 19 (29.2) \\
\hline Angiotensin II Receptor Blocker, n (\%) & $11(16.9)$ \\
\hline Loop Diuretic, n (\%) & $54(83.1)$ \\
\hline Digoxin, n (\%) & $8(12.3)$ \\
\hline Galectin-3, median & $9.82(7.94-12.00)$ \\
\hline ST2, median & $27.22(15.45-44.39)$ \\
\hline NT-proBNP (Admission), median & $5701.0(1867-11961)$ \\
\hline
\end{tabular}

IQR: interquartile range and minimum/maximum, SD: standard deviation, CVD: cardiovascular disease, GFR: glomerular filtration rate, ACE: Angiotensin-Converting-Enzyme.

\section{Univariate Cox analysis}

We acknowledged that Gal-3 $\geq 11.41 \mathrm{ng} / \mathrm{mL}$ correlated with short-term rehospitalization (HR: 11.762, 95\% Cl: 2.40257.598, P-value $=0.002$ ).

A link between Gal-3 $\geq 10.97 \mathrm{ng} / \mathrm{mL}$ and short-term mortality (HR: $13.731,95 \% \mathrm{Cl}: 1.650-114.276$, $\mathrm{P}$ value $=0.015$, Figure 2) and end of follow-up mortality (HR: $3.418,95 \% \mathrm{Cl}$ : 1.345-8.689, P-value $=0.010$ ) was also identified.

The association of NT-proBNP $\geq 21336 \mathrm{ng} / \mathrm{L}$ with Gal-3 $\geq 10.97$ further increased the risk of short-term rehospitalization (HR: $11.985,95 \%$ Cl: 1.962-73.218, $\mathrm{P}$ value $=0.007$ ) and end of follow-up mortality (HR: 78.025, 95\% Cl: 7.592801.926, P-value $<0.001$ ), as shown in Table 2.

A correlation between ST2 $\geq 24.78 \mathrm{ng} / \mathrm{L}$ and end of follow-up mortality was acknowledged (HR: $4.846,95 \% \mathrm{Cl}$ : 1.396-16.825, P-value $=0.013$, Table 3).

If values of NT-proBNP $\geq 21336 \mathrm{ng} / \mathrm{L}$ were added to levels of $\mathrm{ST} 2 \geq 24.78 \mathrm{ng} / \mathrm{L}$ the risk augmented up to 6 times (HR:
5.953, 95\% Cl: $1.683-21.055$, P-value $=0.006)$ and if, instead, values of Gal-3 $\geq 9.99 \mathrm{ng} / \mathrm{mL}$ were considered the risk increased roughly to the same extent (HR: 6.209, 95\% Cl: 2.393-16.114, P-value $<0.001$, Table 3).

For the HFrEF subgroup the end of follow-up mortality risk for the combined values of ST2 $\geq 24.78 \mathrm{ng} / \mathrm{L}$ and Gal-3 $\geq 9.99 \mathrm{ng} / \mathrm{mL}$ was highest (HR: $15.782,95 \% \mathrm{Cl}$ : 1.593156.322, P-value $=0.018$ ) compared to the general population (HR: 6.209, 95\% Cl: 2.393-16.114, P-value $<0.001$ ) and the HFpEF subgroup (HR: 5.2, 95\% Cl: 1.223-22.187, $P$-value $=0.026$, Table 3).

\section{DISCUSSION}

Gal-3 is a soluble $\beta$-galactoside-binding protein which is produced by activated macrophages. It plays a pivotal role in cardiac fibroblast proliferation and collagen synthesis which result in left ventricular stiffness and ultimately in ventricular dysfunction. ${ }^{12}$

Although this acute and chronic inflammation marker is not specific of cardiac injury, since it has been involved in various organs fibrotic degeneration processes ${ }^{13}$ and even with ageing ${ }^{14}$ and tumor mediation ${ }^{15}$, the healthy heart expresses minimum amounts of Gal-3, which increases as HF progresses. ${ }^{16}$

Our finding that patients with renal impairment have higher Gal-3 levels is corroborated by various trials. ${ }^{17,18,19,20}$

We are able to verify the assumption of numerous authors that patients with higher levels of Gal-3 have, also, higher levels of brain natriuretic peptides. $6,17,18,19,20$

In our research no correlation was identified between Gal-3 serum determinations and gender or LVEF, findings reminiscent of those of a sub analysis of the HF-ACTION study. ${ }^{21}$

Concerning NYHA functional class no correlation with Gal-3 was identified by us, likewise the sub analysis of the DEALHF and PRIDE HF trials. ${ }^{11,17,18,22}$

Meijers WC et al. observed an association between elevated plasma Gal-3 and short-term readmission in $\mathrm{HF}^{23}$, which supports our results.

The role of Gal-3 as a predictor of short-term mortality is backed by the PRIDE sub analysis. ${ }^{11}$

The DEAL-HF trial enrolled NYHA functional class III and IV patients, a study population similar to ours, and recognized that Gal-3 was a reliable mortality predictor. ${ }^{18}$

A recent metanalysis also verified a correlation between Gal3 and mortality. ${ }^{24}$

The sub analysis of the $\mathrm{COACH}$ trial, with a follow-up duration similar to ours, verified that Gal-3 was a predictor of the composite of all-cause mortality and HF hospitalization and that a multimarker strategy with NT-proBNP perfected risk stratification, which validates our findings. ${ }^{6}$

We emphasize that mortality risk related to Gal-3 decreased during follow-up which supports the premise that the first 90 
Table 2. Crude survival analysis for Gal-3 $\geq 10.97 \mathrm{ng} / \mathrm{mL}$

\begin{tabular}{|c|c|c|c|}
\hline Biomarker & Early rehospitalization & Early mortality & End of follow-up mortality \\
\hline & $\mathrm{HR}(95 \% \mathrm{Cl})$ & $\mathrm{HR}(95 \% \mathrm{Cl})$ & $\mathrm{HR}(95 \% \mathrm{Cl})$ \\
\hline Gal-3 $\geq 10.97 \mathrm{ng} / \mathrm{mL}$ & $\begin{array}{c}9.886 \\
(2.027-48.214)^{\star \star}\end{array}$ & $\begin{array}{c}13.731 \\
(1.650-14.276)^{\star \star}\end{array}$ & $\begin{array}{c}3.418 \\
(1.345-8.689)^{\star \star}\end{array}$ \\
\hline $\begin{array}{l}\text { Gal-3 } \geq 10.97 \mathrm{ng} / \mathrm{mL} \text { and NT- } \\
\text { proBNP } \geq 21336 \mathrm{ng} / \mathrm{L}\end{array}$ & $\begin{array}{c}11.985 \\
(1.962-73.218)^{\star \star}\end{array}$ & NC & $\begin{array}{c}78.025 \\
(7.592-801.926)^{\star \star \star}\end{array}$ \\
\hline
\end{tabular}

Table 3. End of follow-up mortality crude survival analysis for ST2 $\geq 24.78 \mathrm{pg} / \mathrm{mL}$

\begin{tabular}{|c|c|}
\hline Biomarker & $\begin{array}{l}\text { End of follow-up mortality } \\
\qquad \mathrm{HR}(95 \% \mathrm{Cl})\end{array}$ \\
\hline ST2 $\geq 24.78$ ng/L (population study) & $4.846(1.396-16.825)^{\star \star}$ \\
\hline ST2 $\geq 24.78$ ng/L NT-proBNP $\geq 21336$ ng/L (population study) & $5.953(1.683-21.055)^{\star *}$ \\
\hline ST2 $\geq 24.78$ ng/L Gal-3 $\geq 9.99$ ng/mL (population study) & $6.209(2.393-16.114)^{\star \star \star}$ \\
\hline $\begin{array}{l}\text { ST2 } \geq 24.78 \mathrm{pg} / \mathrm{m} \text { and } \mathrm{Gal}-3 \geq 9.99 \mathrm{ng} / \mathrm{mL} \\
\text { (HFpEF subgroup) }\end{array}$ & $5.2(1.223-22.187)^{\star \star}$ \\
\hline ST2 $\geq 24.78$ ng/L Gal-3 $\geq 9.99$ ng/mL (HFrEF subgroup) & $15.782(1.593-156.322)$ ** \\
\hline
\end{tabular}

HFpEF: Heart Failure with Preserved Ejection Fraction, HFrEF: Heart Failure with Reduced Ejection Fraction.

days post-discharge implicate a maximum risk period that declines over time.

The pathophysiological role of ST2 in HF, is based in the cardioprotective effect of the interaction of its transmembrane receptor (ST2L) and its ligand, IL-33, a cytokine released in response to cardiomyocyte strain. ${ }^{25}$

The duality IL-33/ST2L, participates in several inflammatory conditions, beyond cardiac distress, but although unspecific, it is expressed in cardiac fibroblasts and cardiomyocytes due to myocardial damage. ${ }^{25}$

Its beneficial cardiac effect derives from the counterbalance of cardiac apoptosis, myocardial fibrosis, cardiomyocyte hypertrophy, and ultimately, by increasing myocardial function. ${ }^{25}$ This advantageous effect is abrogated by the soluble isoform (SST2), which acts as a decoy receptor, by binding to IL-33 and blocking its signaling. ${ }^{25}$

Thus, the rationale of using SST2 as HF biomarker relies on the fact that its levels are increased in cardiomyocyte damage, namely remodeling, fibrosis and hypertrophy. ${ }^{25}$

Likewise several authors, we identified that ST2 levels vary inversely with the GFR. ${ }^{7,26,27}$

As for age, as acknowledged by Rehman S et al., ${ }^{7}$ no consistent correlation was identified in our investigation.

Our finding that heightened levels of ST2 predicted end of follow-up mortality is confirmed by the aforementioned study (the AUC ROC for mortality at 1 year was 0.71 ). ${ }^{7}$

This conclusion is, also, sustained by the PRIDE trial, since a trend towards high concentrations of ST2 and one year-mor- tality was acknowledged. ${ }^{28}$

Relevantly, the mutual quantification of ST2 and NT-proBNP improved risk stratification, ${ }^{28}$ as verified in our research. Similarly to Wang $\mathrm{CH}$ et al, ${ }^{29}$ the combination of fibrosis biomarkers identified patients with higher risk of mortality. As expected end of follow-up mortality risk linked to ST2 and Gal-3 simultaneous quantification, was greatest in the HFrEF, which is a more vulnerable subgroup, and lowest in the HFpEF.

The weight of evidence suggests that Gal-3 and ST2 can be useful tools to detect and stratify high risk HF patients.

Given their role in cardiac remodeling and consequently in the $\mathrm{HF}$ inexorable continuum, one must question if in a near future they will ascend as new cardiovascular therapeutic targets.

Due to the small sample size we did not perform multivariable analysis. Moreover, our research was a single-center study which may limit the extrapolation of our acknowledgments. Nevertheless, this study reports data from a real-world clinical background and the results are consistent with previously published large scale studies, therefore supporting the validity of our findings.

\section{CONCLUSIONS}

Elevated Gal-3 concentrations correlated with short-term rehospitalization, short-term mortality and end of follow-up mortality; whereas ST2 prognosticated end of follow-up mortality.

These results corroborate the assumption that promising novel biomarkers Gal-3 and ST2 could be valuable for HF risk stratification. 
We highlight that a multi-marker strategy added information, as a synergism between myocardial fibrosis biomarkers and NT-proBNP was observed.

\section{CONFLICTS OF INTEREST AND SOURCE OF FUNDING}

OM Pharma, Alfragide, Portugal funded the study.

Dr. Mário Barbosa was granted a research scholarship by AstraZeneca and receives fees for lectures from Novartis.

\section{REFERENCES}

1. Vaduganathan M, Fonarow GC, Gheorghiade M. Drug therapy to reduce early readmission risk in heart failure: ready for prime time? JACC Heart Fail. 2013;1(4):361-4.

2. Gheorghiade M, Peterson ED. Improving postdischarge outcomes in patients hospitalized for acute heart failure syndromes. JAMA. 2011;305(23):2456-7.

3. Ravassa S, González A, Bayés-Genís A, et al. Myocardial interstitial fibrosis in the era of precision medicine. Biomarker-based phenotyping for a personalized treatment. Rev Esp Cardiol (Engl Ed). 2020;73(3):248-254.

4-. Daniela Lopes, Luiz Menezes Falcão. Adrenomedulina e ST2 na Insuficiência Cardíaca: Contribuição para Diagnóstico e Prognóstico. Rev Port Cardiol. 2017; 36 (6): 465-472

5. Ana Rita Pereira, Luiz Menezes Falcão. Galectina-3: Indicador de prognóstico. Alvo de intervenção terapêutica? Revista Portuguesa de Cardiologia. 2015; 34 (3): 201208.

6. De Boer RA, Lok DJA, Jaarsma T, et al. Predictive value of plasma galectin-3 levels in heart failure with reduced and preserved ejection fraction. Ann Med. 2011:43:60-8.

7. Rehman S, Mueller T, Januzzi J. Characteristics of the novel interleukin family biomarker ST2 in patients with acute heart failure. J Am Coll Cardiol. 2008;52:1458-65

8. Piper S, deCourcey J, Sherwood R, et al. Biologic Variability of Soluble ST2 in Patients With Stable Chronic Heart Failure and Implications for Monitoring. Am J Cardiol. 2016;118(1):95-8.

9. Yancy CW, Jessup M, Bozkurt B, et al. 2013 ACCF/AHA guideline for the management of heart failure: a report of the American College of Cardiology Foundation/American Heart Association Task Force on Practice Guidelines. J Am Coll Cardiol. 2013; 62: 1495-1539.

10. Ponikowski P, Voors AA, Anker SD, et al; Authors/Task Force Members; Document Reviewers. 2016 ESC Guidelines for the diagnosis and treatment of acute and chronic heart failure: The Task Force for the diagnosis and treatment of acute and chronic heart failure of the European Society of Cardiology (ESC). Developed with the special contribution of the Heart Failure Association (HFA) of the ESC. Eur $J$ Heart Fail. 2016;18(8):891-975.

11. van Kimmenade RR, Januzzi JL Jr, Ellinor PT, et al. Utility of amino-terminal probrain natriuretic peptide, galectin-3, and apelin for the evaluation of patients with acute heart failure. J Am Coll Cardiol. 2006;48:1217-24.
12. Lin YH, Lin LY, Wu YW, et al. The relationship between serum galectin-3 and serum markers of cardiac extracellular matrix turnover in heart failure patients. Clin Chim Acta. 2009;409:96-9

13. Liu FT, Rabinovich GA. Galectins: regulators of acute and chronic inflammation. Ann N Y Acad Sci. 2010;1183:158-82.

14. lacobini C, Amadio L, Oddi G, et al. Role of galectin-3 in diabetic nephropathy. J Am Soc Nephrol. 2003;14:S264-70.

15. Fortuna-Costa A, Gomes AM, Kozlowski EO, et al. Extracellular galectin-3 in tumor progression and metastasis. Front Oncol. 2014:4:138.

16. Rousseau MF, Gruson D, Lepoutre T, et al. Galectin-3 is a strong early predictor of mortality in severe congestive heart failure. Circulation. 2011;124:A10012.

17. Shah RV, Chen-Tournoux AA, Picard MH, et al. Galectin-3, cardiac structure and function, and long-term mortality in patients with acutely decompensated heart failure. Eur J Heart Fail. 2010;12:826-32.

18. Lok DJ, van Der Meer $P$, de la Porte PW, et al. Prognostic value of galectin-3, a novel marker of fibrosis, in patients with chronic heart failure: Data from the DEALHF study. Clin Res Cardiol. 2010;99:323-8.

19. De Boer RA, van Veldhuisen DJ, Gansevoort RT, et al. The fibrosis marker galectin-3 and outcome in the general population. J Intern Med. 2012;272:55-64.

20. De Boer RA, Lok D, Hillege JL, et al. Clinical and prognostic value of Galectin-3, a novel fibrosis-associated biomarker. Relation with clinical and biochemical correlates of heart failure. J Am Coll Cardiol. 2010;55:A26.

21. Felker GM, Fiuzat M, Shaw LK, et al. Galectin-3 in ambulatory patients with heart failure: Results from the HF-ACTION study. Circ Heart Fail. 2012;5:72-8.

22. Ahmad T, Felker GM. Galectin-3 in heart failure: More answers or more questions? J Am Heart Assoc. 2012;1:e004374.

23. Meijers WC, Januzzi JL, deFilippi C, et al. Elevated plasma galectin-3 is associated with near-term rehospitalization in heart failure: a pooled analysis of 3 clinical trials. Am Heart J. 2014;167(6), 853-60.e4.

24. Hongsen Chen, Chensong Chen, Junjie Fang, et al. Circulating galectin-3 on admission and prognosis in acute heart failure patients: a meta-analysis. Heart Failure Reviews. 2020; 25:331-341.

25. Januzzi J, Mebazaa A, Di Somma S. ST2 and prognosis in acutely decompensated heart failure: the International ST2 Consensus Panel. Am J Cardiol. 2015;115:26B-31B.

26. Bayes-Genis A, Zamora E, De Antonio M, et al. Soluble ST2 serum concentration and renal function in heart failure. J Card Fail. 2013;19:768-75.

27. Felker G, Fiuzat M, Thompson V, et al. Soluble ST2 in ambulatory patients with heart failure: association with functional capacity and long-term outcomes. Circ: Heart Fail. 2013; 6:1172-9.

28. Januzzi J, Peacock W, Maisel A, et al. Measurement of the interleukin family member ST2 in patients with acute dyspnea: results from the PRIDE (Pro-Brain Natriuretic Peptide Investigation of Dyspnea in the Emergency Department) study. J Am Coll Cardiol. 2007;50:607-13.

29. Wang $\mathrm{CH}$, Yang NI, Liu MH, et al. Estimating systemic fibrosis by combining galectin-3 and ST2 provides powerful risk stratification value for patients after acute decompensated heart failure. Cardiol J. 2016; 23(5):563-572. 